Schmerz 2016 $30: 383-385$

DOI 10.1007/s00482-016-0165-y

Online publiziert: 28. September 2016

(c) Deutsche Schmerzgesellschaft e.V. Published by Springer-Verlag Berlin Heidelberg - all rights reserved 2016

CrossMark

\author{
M. I. Hasenbring ${ }^{1} \cdot$ R. Klinger ${ }^{2} \cdot$ K. Thieme ${ }^{3}$ \\ 'Dept. of Medical Psychology and Medical Sociology, Faculty of Medicine, Ruhr-University of Bochum, \\ Bochum, Deutschland \\ ${ }^{2}$ Zentrum für Anästhesiologie und Intensivmedizin Klinik und Poliklinik für Anästhesiologie, UKE, \\ Hamburg, Deutschland \\ ${ }^{3}$ Institut für Medizinische Psychologie, Philipps-Universität Marburg, Marburg, Deutschland
}

\title{
Schmerzpsychologie interdisziplinär I: Psychobiologische Risikofaktoren und Mechanismen
}

gehen, einwirken. Allerdings beginnen wir erst damit, das „Wie“, d.h. die konkreten Mechanismen psychobiologischer Wechselwirkungen - in beide Wirkungsrichtungen - zu verstehen. Wir entdecken darüber hinaus, dass die beteiligten psychischen Faktoren weit vielfältiger sind als bisher angenommen. Ihr Zusammenspiel und ihre Wirkung auf das Schmerzerleben sind nicht immer eindeutig günstig oder ungünstig. Dies trifft nicht nur auf bereits chronifizierte Schmerzerkrankungen zu, sondern auch auf akute Schmerzen.

Der vorliegende Band I gibt einen Einblick in die aktuellen Forschungsrichtungen zahlreicher deutschsprachiger Arbeitsgruppen zu psychobiologischen Risikofaktoren und Mechanismen, die in der Aufrechterhaltung von Schmerzerkrankungen eine Rolle spielen. Der erste Beitrag beschäftigt sich mit dem Phänomen der Placeboreaktion, welches im Rahmen einer großen, bundesweiten Forschungsinitiative untersucht wird und das unmittelbare Schmerzerleben in den Mittelpunkt ihrer Forschung rückt. Am Beispiel experimenteller aber auch klinischer Schmerzen und Juckreizphänomene beschreibt A. Sölle mit ihren Koautorinnen [1] vor allem Lern- und Erwartungsprozesse, die einer wirkstofffreien Substanz die Wirksamkeit eines Medikamentes verleihen und auch die Effektivität eines Medikamentes steigern können. Sie plädieren für eine gezielte, ethische Richtlinien berücksichtigende Nutzung strukturierter Placeboreaktionen. Eine solche Nutzung sollte nicht nur im Rahmen der medikamentösen Therapie zum Einsatz kommen - und in jedem Fall eine Schulung der Kommunikation mit dem Patienten zur Voraussetzung haben. Becker und Diers [2] beleuchten in ihrem Beitrag die Rolle von Belohnungsreizen, die im Alltag häufig mit schmerzinkompatiblen Aktivitäten verknüpft sind und den Betroffenen mit einem Konflikt konfrontieren: soll dem Schmerz oder aber der Belohnung Priorität in der Aufmerksamkeit gelten? Liegt bei Gesunden ein solcher Konflikt vor, kann dies, falls die Entscheidung für die Belohnung ausfällt, eine endogene Schmerzhemmung auslösen. Bei Patienten mit chronischen muskuloskelettalen oder neuropathischen Schmerzen scheint diese Hemmung sich dagegen nicht oder nur eingeschränkt einzustellen, sie weisen verschiedene Zeichen einer dysfunktionalen Belohnungsverarbeitung auf. Hierfür sprechen erste verhaltensbezogene sowie neurale Bildgebungsdaten. Elsenbruch und Enck [3] zeigen in ihrem Überblicksbeitrag auf, in welchem Ausmaß psychobiologische Mechanismen, die in der Aufrechterhaltung muskuloskelettaler oder neuropathischer Schmerzen bisher nachgewiesen wurden, auch bei viszeralen Schmerzerkrankungen eine zentrale Rolle spielen. Am Beispiel des Reizdarmsyndroms lem mit chronischen Schmerzen einher- 
erläutern sie den aktuellen Stand der Forschung, der in jüngeren Jahren neben akutem und chronischem Stress, negativen Emotionen auch die Placeboreaktion gezielt untersucht. Negative Emotionen und Ihre Regulierung, dabei die neuerdings zunehmend beachtete Vielfalt möglicher Emotionsregulationsstrategien werden in dem Beitrag von Konietzny, Chehadi und KoautorInnen [4] eingehender beleuchtet. Basierend auf der Kenntnis hoher Komorbidität chronischer Schmerzerkrankungen mit psychopathologischen Störungen, insbesondere aus dem affektiven Formenkreis, schlagen die AutorInnen einen transdiagnostischen Ansatz vor, der die gemeinsamen Anteile chronischer Schmerzen und affektiver Störungen untersucht. Sie beleuchten dabei vor allem verhaltensbezogene und neuronale Befunde zur Wirkung einzelner auch bisher wenig erforschter Strategien der Emotionsregulation und damit die wissenschaftliche Basis für transdiagnostische kognitivvehaltenstherapeutische Ansätze.

Beziehen sich die ersten vier Beiträge dieses Heftes primär auf die Rolle kognitiv-affektiver und lernpsychologischer Mechanismen, so wenden sich die nächsten drei Beträge schwerpunktmäßig körperlichen Aspekten zu: körperlicher Aktivität vor allem bei muskuloskelettalen Schmerzen, den Mechanismen der Adaptivität an körperliche Aktivität und Stress sowie der Rolle des Körperbildes bei Patienten mit chronischem Schmerz. Titze, Gajsar und Koautorinnen [5] machen mit ihrem Fokus-Review aufmerksam auf die Komplexität des $\mathrm{Zu}$ sammenhanges zwischen körperlicher Aktivität und Schmerz, bei dem wir nicht von einem "Je mehr, desto besser", also einem linear positiven Zusammenhang ausgehen können. Basierend auf zahlreichen klinischen und experimentellen Forschungsansätzen wird deutlich gemacht, dass die Wirkung körperlicher Aktivität auf den Organismus abhängig ist von verschiedenen Kontextfaktoren, u. a. ob es sich um gezielt sportliche, berufliche oder alltagsbezogene Aktivitäten geht. Zudem kann körperliche Aktivität, auch sportlicher Art, im Falle chronischer Schmerzen dysfunktionale Effekte entfalten und nicht zuletzt, ist ein dysfunktional zu niedriges aber auch ein zu hohes Maß an körperlicher Aktivität mit Mustern dysfunktionaler Schmerzverarbeitung assoziiert. Psychologische und neurobiologische Prozesse der Adaptivität an körperliche, insbesondere sportliche Aktivität werden im Beitrag von Wippert und Wiebking [6] erläutert, die für die Erarbeitung optimaler DosisWirkungsbeziehungen und ihrer Determinanten plädieren, die die Effektivität bewegungsorientierter Therapieverfahren erhöhen können. Schließlich zeigen Levenig und KoautorInnen in ihrem Überblick auf, dass Merkmale einer veränderten Körperwahrnehmung und eines veränderten Körperbildes an der Aufrechterhaltung chronischer Schmerzen, hier vor allem bezogen auf den chronischen Rückenschmerz, beteiligt sein können. Dabei sind mögliche Ursache-Wirkungsrichtungen noch sehr wenig erforscht [7].

Soziale Interaktionen, hier insbesondere Merkmale habituellen Bindungsverhaltens untersuchen Pfeifer, Neubauer und KoautorInnen hinsichtlich ihres Zusammenhanges mit chronischen Schmerzen, schwerpunktmäßig aus dem Bereich somatoformer Schmerzstörungen [8]. Vor allem ängstlich-anklammernde und ängstlich-meidende Bindungsmuster sind assoziiert mit dysfunktionalen Schmerzverarbeitungsformen, u. a. dem Katastrophisieren, mit besonders häufigen Arztkontakten sowie mit vermehrter Opioideinnahme. In einem zweiten Beitrag erläutern sie die Rolle des körpereigenen Hormons Oxytocin (OT), oft auch als Bindungshormon bezeichnet, für das menschliche Schmerzerleben. Patienten mit chronischen Schmerzerkrankungen scheinen einen geringeren OT-Spiegel aufzuweisen als Gesunde [9]. In einer systematischen Analyse von 17 experimentellen Studien finden sich erste Hinweise für analgetische Effekte von $\mathrm{OT}$, für erhöhte viszerale Wahrnehmungsschwellen, einen deutlicheren Placeboeffekt wie auch verminderte Amygdala-Aktivität unter Schmerzreizung in der Bildgebung, eine Region, die für die Verarbeitung negativer Emotionen steht. Ein neurophysiologischer Mechanismus, der Baroreflex, steht im Mittelpunkt des abschließenden Beitra- ges dieses Sonderbandes und beleuchtet die Interaktion des kardiovaskulären Systems mit der Schmerzwahrnehmung und -verarbeitung. Meller und KoautorInnen zeigen auf, das Patienten mit chronischen Schmerzen, vor allem, wenn psychologische Risikofaktoren am Schmerzgeschehen beteiligt sind, eine verminderte Baroreflexsensitivität aufweisen, die sie in Zusammenhang mit einer fehlenden oder verminderten endogenen Schmerzhemmung infolge klassischer und operanter Konditionierung auf biologischer Ebene diskutieren [10].

Mit der Vielfalt der hier beschriebenen psychobiologischen Wechselwirkungen, den zahlreichen neuen psychologischen Merkmalen, die jüngst betrachtet werden aber auch dem besseren Verständnis schmerzrelevanter Mechanismen ergeben sich eine Vielzahl von Ansatzpunkten, die schmerzpsychologischen Therapieverfahren zu optimieren, sie anzureichern, zu konkretisieren und nicht zuletzt, ihre Wirkungsweise besser zu verstehen. Davon wird nicht nur ihre Effektivität sondern auch ihre Vermittelbarkeit gegenüber nichtpsychologischen Behandlern und gegenüber dem Patienten profitieren.

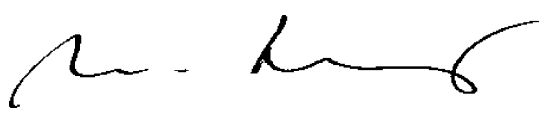

M.I. Hasenbring

\section{Korrespondenzadresse}

\section{Prof. Dr. M. I. Hasenbring}

Dept. of Medical Psychology and Medical Sociology, Faculty of Medicine, Ruhr-University of Bochum

Universitätsstr. 150, Bochum, Deutschland Monika.Hasenbring@rub.de

Interessenkonflikt. M. I. Hasenbring, R. Klinger und K. Thieme geben an, dass kein Interessenkonflikt besteht.

\section{Literatur}

1. Sölle A, Worm M, Flor H, Klinger R (2016) Placeboreaktion. Mechanismen und klinische Anwendungen. Schmerz. doi:10.1007/s00482016-0138-1

2. Becker S, Diers M (2016) Chronischer Schmerz. Wahrnehmung, Belohnung und neurale Ver- 
arbeitung. Schmerz. doi:10.1007/s00482-0160149-y

3. Elsenbruch S, Enck P (2016) Psychobiologische Mechanismen bei der Pathophysiologie chronischer viszeraler Schmerzen. Schmerz. doi:10.1007/ s00482-016-0130-9

4. Konietzny K, Suchan B, Kreddig N, Hasenbring Ml, Chehadi O (2016) Emotionsregulation und Schmerzen. Behaviorale und neuronale Korrelate - ein transdiagnostischer Ansatz. Schmerz. doi:10. 1007/s00482-016-0162-1

5. Titze C, Gajsar H, Hasenbring MI (2016) Körperliche Aktivität und muskuloskeletale Schmerzen. Ein Fokus-Review aus dem MiSpEx-Forschungsverbund. Schmerz. doi:10.1007/s00482-016-0152-3

6. Wippert P-M, Wiebking C (2016) Adaptation an körperliche Aktivität und psychischen Stress im Kontext von Schmerz. Schmerz. doi:10.1007/ s00482-016-0147-0

7. Levenig CG, Hasenbring MI, Kleinert J, Kellmann M (2016) Körperbild und Rückenschmerz. Schmerz. doi:10.1007/s00482-016-0122-9

8. Pfeifer A-C, Ehrenthal JC, Neubaruer E, Gerick C, SchiltenwolfM(2016) Einfluss des Bindungsverhalten auf chronischen und somatoformen Schmerz. Schmerz.doi:10.1007/s00482-016-0156-z

9. Pfeifer A-C, Ditzen B, Neubauer E, Schiltenwolf M (2016) Wirkung von Oxytocin auf das menschliche Schmerzereleben. Schmerz. doi:10.1007/s00482016-0164-z

10. Meller T, Stiehm F, Malinowski R, Thieme K (2016) Baroreflexsensitivität und chronischer Schmerz: pathogenetische Mechanismen und klinische Implikationen. Schmerz. doi:10.1007/s00482016-0150-5

\section{Chronischer lumbaler Rückenschmerz}

Vergleich zwischen Mobilisationstraining und Training der rumpfstabilisierenden Muskulatur

In der Behandlung chronischer lumbaler Rückenschmerzen werden Übungen zur Kräftigung der rumpfstabilisierenden Muskulatur und zur Mobilisation des Rumpfes eingesetzt. Das Ziel der prospektiven randomisierten klinischen Pilotstudie war, die Wirksamkeit einer Trainingsmethode der segmentalen Beweglichkeit der Wirbelsäule mit einem Training der rumpfstabilisierenden Muskulatur hinsichtlich objektiver und subjektiver Outcomes bei Patienten mit chronischen lumbalen Rückenschmerzen zu vergleichen.

Nach einer Per-Protocol-Analyse wurden

die Daten von 27 Patienten mit chronischen lumbalen Rückenschmerzen, die einer von 2 Gruppen randomisiert zugeordnet wurden, ausgewertet. Die Interventionsgruppe führte ein Mobilisationstraining nach der BALLance-Methode ${ }^{\circledR}$, die Vergleichsgruppe ein Training der rumpfstabilisierenden Muskulatur über einen Zeitraum von 4 Wochen mit anschließendem Heimübungsprogramm über weitere 4 Wochen durch. Zu 3 Zeitpunkten (Vorher, nach 4 Wochen, Follow-up nach 8 Wochen) wurden die Beweglichkeit, die Kraftausdauer der Rumpfmuskulatur, die schmerzbedingte Behinderung bei Aktivitäten im Alltag, sowie das Schmerzvermeidungsverhalten erhoben. Im Mittel verbesserte nur die Vergleichsgruppe signifikant die Haltezeit im Unterarmstütz und Seitstütz über die Zeit $(p<0,001)$ mit signifikanten Unterschieden zur Interventionsgruppe $(p<0,05)$. In der Mehrzahl der subjektiven Outcomes konnte innerhalb beider Gruppen eine signifikante Verbesserung festgestellt werden $(p<0,05)$. Zwischen den Gruppen bestanden dabei keine signifikanten Unterschiede.

Patienten mit chronischen lumbalen Rückenschmerzen scheinen in Bezug auf ihre Beschwerden im Alltag sowohl mit gezielten Mobilisationsübungen als auch mit Übungen zur Kräftigung der rumpfstabilisierenden Muskulatur behandelt werden zu können.
Lesen Sie die vollständige Originalie in M.Alfuth, D. Cornely (2016) Chronischer lumbaler Rückenschmerz. Vergleich zwischen Mobilisationstraining und Training der rumpfstabilisierenden Muskulatur. Der Orthopäde 45:579-590 oder als registrierter Nutzer kostenfrei auf Springer Medizin unter www.springermedizin.de/link/8112128

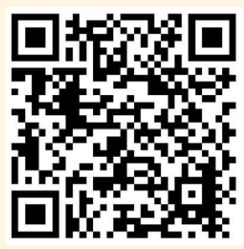

Suchen Sie noch mehr zum Thema? Mit e.Med, dem Online-Paket von Springer Medizin, können Sie schnell und komfortabel in über 500 medizinischen Fachzeitschriften recherchieren.

Weitere Infos unter: springermedizin.de/eMed. 